Research Article

\title{
Reduced-Order Observers for Nonlinear State Estimation in Flexible Multibody Systems
}

\author{
Ilaria Palomba $\mathbb{D D}^{1},{ }^{1}$ Dario Richiedei $\mathbb{D}^{2},{ }^{2}$ and Alberto Trevisani $\mathbb{D}^{2}$ \\ ${ }^{1}$ Faculty of Science and Technology, Free University of Bolzano-Bozen, Piazza Università 5, Bolzano 39100, Italy \\ ${ }^{2}$ Department of Management and Engineering (DTG), Università degli Studi di Padova, Stradella San Nicola 3, \\ Vicenza 36100, Italy \\ Correspondence should be addressed to Alberto Trevisani; alberto.trevisani@unipd.it
}

Received 10 August 2018; Accepted 8 October 2018; Published 1 November 2018

Academic Editor: Chao Tao

Copyright (c) 2018 Ilaria Palomba et al. This is an open access article distributed under the Creative Commons Attribution License, which permits unrestricted use, distribution, and reproduction in any medium, provided the original work is properly cited.

\begin{abstract}
Modern control schemes adopted in multibody systems take advantage of the knowledge of a large set of measurements of the most important state variables to improve system performances. In the case of flexible-link multibody systems, however, the direct measurement of these state variables is not usually possible or convenient. Hence, it is necessary to estimate them through accurate models and a reduced set of measurements ensuring observability. In order to cope with the large dimension of models adopted for flexible multibody systems, this paper exploits model reduction for synthesizing reduced-order nonlinear state observers. Model reduction is done through a modified Craig-Bampton strategy that handles effectively nonlinearities due to large displacements of the mechanism and through a wise selection of the most important coordinates to be retained in the model. Starting from such a reduced nonlinear model, a nonlinear state observer is developed through the extended Kalman filter (EKF). The method is applied to the numerical test case of a six-bar planar mechanism. The smaller size of the model, compared with the original one, preserves accuracy of the estimates while reducing the computational effort.
\end{abstract}

\section{Introduction}

Flexible-link multibody (FLMB) systems are highly promising from an economical and sustainability point of view because of the use of less material and the need for smaller actuators and less power consumption. However, flexibility often results in unwanted vibrations that limit motion accuracy and imposes advanced control schemes accounting for the flexible dynamics. High-performance control schemes are typically model-based [1-5] and often require the knowledge of a large set of state variables [3-5]. For example, a common application requiring the whole state vector (i.e., position and speed of all the state variables) is active control through state feedback to assign the desired modal characteristics $[6,7]$. The knowledge of the tip position of open-chain robots is also needed for precise control of the end-effector trajectory [8]. In contrast, the direct measurement of all the state variables is rarely feasible or convenient [9], so the unmeasured state variables should be estimated by means of state observers that exploit a smaller set of measurements, ensuring the system observability and an accurate system model. Most of the contributions in the literature have been focused on the state estimation in rigidbody multibody systems [10-12], in flexible structures [13] or in single-link flexible mechanisms [2, 14-17]. Complexity is significantly exacerbated in the case of multilink flexible systems where state estimation is, to date, still a challenging task, mainly because of the complex and large dimensional models used to represent these systems that make cumbersome the synthesis of observers.

In the past, due to limitations of the computational power of computers, the use of linearized [18] or linearized reduced-order models has been proved to be a good tradeoff between accuracy and computational complexity [1820]. Indeed, computational effort should be reduced by keeping model size as small as possible to perform real-time estimations. Hence, model reduction is a powerful tool to boost the use of state observers, by representing through the model just the state variables that are of interest for estimation and control purposes (i.e., those having higher 
observability and controllability [20]). Several approaches to model reduction have been presented in the literature to date for linear mechanical systems [21]. For example, in the field of structural dynamics, one of the most widespread reduction techniques is the Craig-Bampton (CB) method [22] because of its effectiveness and ease of implementation.

In the very recent years, the use of nonlinear state observers has been proved to be effective for improving accuracy of state estimation in multibody systems, compared to linearized observers [2]. Indeed, the use of nonlinear models and variable gains of the observer handles more effectively the nonlinear behavior of these systems. This increase of the computational complexity of these observers still calls for the use of reduced-order models.

The typical implementation of the aforementioned $\mathrm{CB}$ method for reducing FLMB systems exploits the component mode synthesis strategy [21]. Basically, the linear FE models of all the links are generated and reduced and, then, they are embedded in a moving frame [23] or in a corotational frame [24], to represent large displacements of the mechanism. Such a component-level reduction strategy might negatively affect the reduced model correctness since it does not fully account for modal characteristic changes due to the motion of the multibody system. To overcome this issue, some studies have recently suggested the use of model reduction at the system-level, i.e., of the model of the whole system. On the one hand, this choice represents more effectively the change of the modal characteristics of the system [25]. On the other hand, system-level reduction ensures a more effective selection of the state variables to be retained in the reduced-order model, thus providing a better trade-off between model size and accuracy [26].

By taking advantage of the idea of using reduced-order nonlinear models in the design of nonlinear observers, this work proposes a novel and comprehensive approach for efficient and accurate state estimation in FLMB systems. The method exploits the modified nonlinear $\mathrm{CB}$ reduction suitable for flexible-link mechanisms based on the equivalent rigid-link system (ERLS) and formulated through ordinary differential equations (and hence independent coordinates) outlined in [25] and a wise selection of the most important coordinates, as proposed in [26]. Such a model, that ensures that the dynamics with the highest observability and controllability are modeled in the system, is used for the synthesis of an extended Kalman filter (EKF) [27] to deliver accurate estimates of both the large motion and of the elastic vibrations of a FLMB system by means of a small set of sensed signals and with a reduced computational effort.

The method is validated numerically, by investigating sensitivity to model uncertainty and measurement noise, by means of a planar 6-link FLMB system.

\section{Modeling}

2.1. Full-Order Dynamic Model: ERLS Approach. In this work, the equations of motion for a FLMB system undergoing large rigid-body motion are modeled through the equivalent rigid-link system (ERLS) formulation. FLMB systems with scleronomous and holonomic constraints are assumed. The motion of the system is notionally separated into the large motion of a rigid-link moving reference configuration (ERLS) and the small elastic deformation of the flexible links with respect to the ERLS itself. Therefore, the configuration of a FLMB system is defined through a set of generalized rigidbody coordinates $\mathbf{q}$ and elastic coordinates $\mathbf{u}$ that are the fullorder model independent coordinates:

$$
\mathbf{x}=\left\{\begin{array}{l}
\mathbf{q} \\
\mathbf{u}
\end{array}\right\},
$$

where the dimension of $\mathbf{x}$, denoted as $n$, is the number of degrees of freedom (dofs) of the full-order model.

The system model is given by the following set of nonlinear ODEs [23]:

$$
\begin{aligned}
& \underbrace{\left[\begin{array}{cc}
\mathbf{S}^{T}(\mathbf{q}) \mathbf{M}(\mathbf{q}) \mathbf{S}(\mathbf{q}) & \mathbf{S}^{T}(\mathbf{q}) \mathbf{M}(\mathbf{q}) \\
\mathbf{M}(\mathbf{q}) \mathbf{S}(\mathbf{q}) & \mathbf{M}(\mathbf{q})
\end{array}\right]}_{\overline{\mathbf{M}}(\mathbf{q})}\left\{\begin{array}{c}
\ddot{\mathbf{q}} \\
\ddot{\mathbf{u}}
\end{array}\right\} \\
& +\underbrace{\left[\begin{array}{cc}
\mathbf{S}^{T}(\mathbf{q}) \mathbf{M}(\mathbf{q}) \dot{\mathbf{S}}(\mathbf{q}, \dot{\mathbf{q}}) & \mathbf{S}^{T}(\mathbf{q})\left(2 \mathbf{M}_{\mathrm{G}}(\mathbf{q})+\mathbf{C}(\mathbf{q})\right) \\
\mathbf{M}(\mathbf{q}) \dot{\mathbf{S}}(\mathbf{q}, \dot{\mathbf{q}}) & 2 \mathbf{M}_{\mathrm{G}}(\mathbf{q})+\mathbf{C}(\mathbf{q})
\end{array}\right]}_{\overline{\mathbf{C}}(\mathbf{q})}\left\{\begin{array}{c}
\dot{\mathbf{q}} \\
\dot{\mathbf{u}}
\end{array}\right\} \\
& +\underbrace{\left[\begin{array}{cc}
\mathbf{0} & \mathbf{0} \\
\mathbf{0} & \mathbf{K}(\mathbf{q})
\end{array}\right]}_{\overline{\mathbf{K}}(\mathbf{q})}\left\{\begin{array}{l}
\mathbf{q} \\
\mathbf{u}
\end{array}\right\}=\underbrace{\left[\begin{array}{cc}
\mathbf{S}^{T}(\mathbf{q}) & \mathbf{S}^{T}(\mathbf{q}) \mathbf{M}(\mathbf{q}) \\
\mathbf{I} & \mathbf{M}(\mathbf{q})
\end{array}\right]\left\{\begin{array}{c}
\mathbf{f}(\mathbf{q}) \\
\mathbf{g}(\mathbf{q})
\end{array}\right\}}_{\overline{\mathbf{F}}(\mathbf{q})}
\end{aligned}
$$

Finite elements are used in Equation (2) to get the mass, stiffness, damping, and centrifugal and Coriolis matrices (denoted $\mathbf{M}, \mathbf{K}, \mathbf{C}$, and $\mathbf{M}_{\mathrm{G}}$, respectively) by assembling the consistent matrices of each finite element. In Equation (2), $\mathbf{g}$ and $\mathbf{f}$ denote the gravity acceleration and the external force vector, respectively. $\mathbf{S}$ is the ERLS sensitivity coefficient matrix for all the nodes of the finite elements and $\dot{\mathbf{S}}$ is its time derivative. The mass, damping, and stiffness matrices of the full-order model have been introduced in Equation (2) and denoted as $\overline{\mathbf{M}}, \overline{\mathbf{C}}$, and $\overline{\mathbf{K}}$, respectively. $\overline{\mathbf{F}}$ is the full-order vector of the external forces.

\subsection{Reduced-Order Dynamic Model: Modified Craig-} Bampton Method. A dynamic model reduced through the $\mathrm{CB}$ method is represented through a hybrid set of coordinates, including some physical coordinates $\mathbf{x}_{m}$ (named the master dofs) and some nonphysical coordinates $\boldsymbol{\eta}$ (named interior modal coordinates).

The full-order set of physical coordinates $\mathbf{x}$ of the original model is approximated through a reduced set of hybrid coordinates $\mathbf{p}$ by means of the CB transformation matrix $\mathbf{H}$ :

$$
\begin{aligned}
& \mathbf{x} \approx \mathbf{H} \cdot \mathbf{p}, \\
& \mathbf{x} \in \mathbb{R}^{n}, \\
& \mathbf{p} \in \mathbb{R}^{r}, \quad r \ll n .
\end{aligned}
$$

In the standard formulation of the CB method, usually adopted for structures and linear systems, the transformation matrix in Equation (3) is constant. Conversely, in the case of 
mechanisms performing large displacements, it should be reformulated as a configuration-dependent matrix to cope with the nonlinearities of the FLMB models [25]:

$$
\begin{aligned}
& \mathbf{x} \approx \mathbf{H}\left(\mathbf{q}, \mathbf{q}^{*}\right) \mathbf{p}, \\
& \mathbf{x}=\left\{\begin{array}{c}
\mathbf{x}_{m} \\
\mathbf{x}_{s}
\end{array}\right\} \in \mathbb{R}^{n=m+s}, \\
& \mathbf{p}=\left\{\begin{array}{c}
\mathbf{x}_{m} \\
\mathbf{\eta}
\end{array}\right\} \in \mathbb{R}^{r=m+p}, \quad p \ll s .
\end{aligned}
$$

In Equation (4), $\mathbf{x}_{m}^{T}=\left\{\begin{array}{ll}\mathbf{q}^{T} & \mathbf{u}_{m}^{T}\end{array}\right\} \in \mathbb{R}^{m}$ is the $m$-dimensional set of the master dofs, which comprises all the ERLS coordinates $\mathbf{q}$ and some meaningful elastic coordinates $\mathbf{u}_{m}$ (e.g., those where external loads are applied). The $s$-dimensional vector $\mathbf{x}_{s}^{T}=\left\{\mathbf{u}_{s}^{T}\right\} \in \mathbb{R}^{s}(m+s=n)$ includes the remaining elastic coordinates that are transformed into interior modal coordinates through the transformation matrix $\mathbf{H}$. The configuration-dependent $\mathrm{CB}$ reduction matrix in Equation (4) is defined as follows:

$$
\mathbf{H}\left(\mathbf{q}, \mathbf{q}^{*}\right)=\left[\begin{array}{cc}
\mathbf{I} & \mathbf{0} \\
\mathbf{B}(\mathbf{q}) & \widetilde{\mathbf{\Psi}}\left(\mathbf{q}, \mathbf{q}^{*}\right)
\end{array}\right] .
$$

In Equation (5), I and $\mathbf{0}$ are the identity and null matrices, respectively, B is the position-dependent Guyan's matrix and $\widetilde{\Psi}$ is the configuration-dependent interior mode matrix. The definition of matrix $\widetilde{\boldsymbol{\Psi}}=\widetilde{\boldsymbol{\Psi}}\left(\mathbf{q}, \mathbf{q}^{*}\right)$ is a key point of the method and is obtained by the following three steps:

(1) Computation of the Interior Mode Matrix $\boldsymbol{\Phi}$ at an Equilibrium Configuration $\mathbf{q}^{*}$. The so-called interior mode matrix $\Phi$ of the subsystem made by the slave dofs is computed through local modal analysis. Since the model is nonlinear, modal analysis is done by placing the ERLS at a given equilibrium configuration $\mathbf{q}^{*}$ (obtained by setting $\dot{\mathbf{q}}=\ddot{\mathbf{q}}=\mathbf{0}$ ) and linearizing the system with the master dofs constrained. The equilibrium point can be conveniently chosen as any arbitrary point belonging to the desired trajectory.

(2) Selection of the Interior Modes. A suitable number of columns of $\Phi$ is removed to get the truncated interior mode matrix $\widetilde{\boldsymbol{\Phi}}$. Such a rectangular matrix approximates the full-order set of slave dofs with a smaller set of meaningful interior coordinates (Equation (4)). A key point for getting accurate reduced models is the proper selection of the interior modes to be retained $[26,28,29]$. In Section 4.1, the strategy followed in this paper will be briefly explained, even if any arbitrary selection method can be adopted.

(3) Definition of the Configuration-Dependent Interior Mode Matrix $\widetilde{\boldsymbol{\Psi}}$. The truncated interior mode matrix $\widetilde{\boldsymbol{\Phi}}$, computed for $\mathbf{q}=\mathbf{q}^{*}$, should be transformed to follow the motion of the ERLS. Under the reasonable hypothesis of small elastic deformations with respect to the ERLS, on which the ERLS theory is based, eigenmodes change slowly if expressed with respect to the reference system of the finite elements. Such an idea is schematically represented in Figure 1, where for sake of clarity a planar single flexible link has been represented. In the ERLS model, the components of each eigenvector $\varphi$ are expressed with respect to the ERLS configurations (Figures 1(a) and 1(b)), and it is evident that these components are different. However, if a local reference frame $\{\mathbf{x}, \mathbf{y}\}$ is adopted to express the eigenvectors, the coordinates are almost the same also for different ERLS configurations (Figures 1(c) and 1(d)). This hypothesis has been assessed in [25]. Therefore, it is possible to extend the validity of the interior mode matrix $\widetilde{\boldsymbol{\Phi}}$, computed at $\mathbf{q}^{*}$, in a wide subset of the workspace, by projecting it onto the local reference frame of each finite elements and then projecting it again onto the actual instantaneous ERSL $\mathbf{q}$ through a suitable transformation matrix $\mathbf{R}=$ $\mathbf{R}\left(\mathbf{q}, \mathbf{q}^{*}\right)$ (see [25] for a detailed description):

$$
\widetilde{\boldsymbol{\Psi}}\left(\mathbf{q}, \mathbf{q}^{*}\right)=\mathbf{R}\left(\mathbf{q}, \mathbf{q}^{*}\right) \widetilde{\boldsymbol{\Phi}}\left(\mathbf{q}^{*}\right) \text {. }
$$

Once $\mathbf{H}$ is defined, the reduced model is obtained by premultiplying Equation (2) by $\mathbf{H}^{T}$ :

$$
\begin{aligned}
& \underbrace{\mathbf{H}^{T}\left(\mathbf{q}, \mathbf{q}^{*}\right) \overline{\mathbf{M}}(\mathbf{q}) \mathbf{H}\left(\mathbf{q}, \mathbf{q}^{*}\right)}_{\widetilde{\mathbf{M}}}\left\{\begin{array}{c}
\ddot{\mathbf{x}}_{m} \\
\ddot{\boldsymbol{\eta}}
\end{array}\right\}+\underbrace{\mathbf{H}^{\mathrm{T}}\left(\mathbf{q}, \mathbf{q}^{*}\right) \overline{\mathbf{C}}(\mathbf{q}, \dot{\mathbf{q}}) \mathbf{H}\left(\mathbf{q}, \mathbf{q}^{*}\right)}_{\widetilde{\mathbf{C}}}\left\{\begin{array}{c}
\dot{\mathbf{x}}_{m} \\
\dot{\boldsymbol{\eta}}
\end{array}\right\} \\
& +\underbrace{\mathbf{H}^{T}\left(\mathbf{q}, \mathbf{q}^{*}\right) \overline{\mathbf{K}}(\mathbf{q}) \mathbf{H}\left(\mathbf{q}, \mathbf{q}^{*}\right)}_{\widetilde{\mathbf{K}}}\left\{\begin{array}{c}
\mathbf{x}_{m} \\
\boldsymbol{\eta}
\end{array}\right\}=\underbrace{\mathbf{H}^{T}\left(\mathbf{q}, \mathbf{q}^{*}\right) \overline{\mathbf{F}}(\mathbf{q})}_{\widetilde{\mathbf{F}}} .
\end{aligned}
$$

The mass, damping, and stiffness matrices of the reduced model have been introduced in Equation (7) and denoted as $\widetilde{\mathbf{M}}, \widetilde{\mathbf{C}}$, and $\widetilde{\mathbf{K}}$ respectively. $\widetilde{\mathbf{F}}$ is the reduced-order vector of the external forces.

\section{State Estimation}

3.1. Model Formulation. The synthesis of a state observer requires the dynamic model in Equation (7) to be formulated in a state-space (first-order) form, as follows:

$$
\left\{\begin{array}{l}
\dot{\mathbf{z}}=f(\mathbf{z}, \widetilde{\mathbf{F}}), \\
\mathbf{y}=g(\mathbf{z}, \widetilde{\mathbf{F}}),
\end{array}\right.
$$

where $\mathbf{z}=\left\{\begin{array}{ll}\mathbf{p}^{T} & \dot{\mathbf{p}}^{T}\end{array}\right\}^{T}$ is the reduced-order state vector, $\dot{\mathbf{z}}$ is its derivative, and $\mathbf{y}$ is the vector of the measured outputs (often denoted as the observations). Both the system equation $f$ and the measurement equation $g$ in Equation (8) are usually nonlinear functions of the state and of the input $\widetilde{\mathbf{F}}$. The direct use of ODEs for the formulation of the multibody model in Equation (2) makes straightforward the achievement of the state-space form required for state estimation.

By taking advantage of Equation (7), the nonlinear continuous-time system equations in Equation (8) take the following form:

$$
\begin{aligned}
\dot{\mathbf{z}}= & \left\{\begin{array}{l}
\dot{\mathbf{p}} \\
\ddot{\mathbf{p}}
\end{array}\right\}=\left[\begin{array}{cc}
0 & \mathbf{I} \\
-\widetilde{\mathbf{M}}^{-1}(\mathbf{q}) \widetilde{\mathbf{K}}(\mathbf{q}) & -\widetilde{\mathbf{M}}^{-1}(\mathbf{q}) \widetilde{\mathbf{C}}(\mathbf{q}, \dot{\mathbf{q}})
\end{array}\right]\left\{\begin{array}{l}
\mathbf{p} \\
\dot{\mathbf{p}}
\end{array}\right\} \\
& +\left[\begin{array}{c}
0 \\
\widetilde{\mathbf{M}}^{-1}(\mathbf{q})
\end{array}\right] \widetilde{\mathbf{F}} .
\end{aligned}
$$




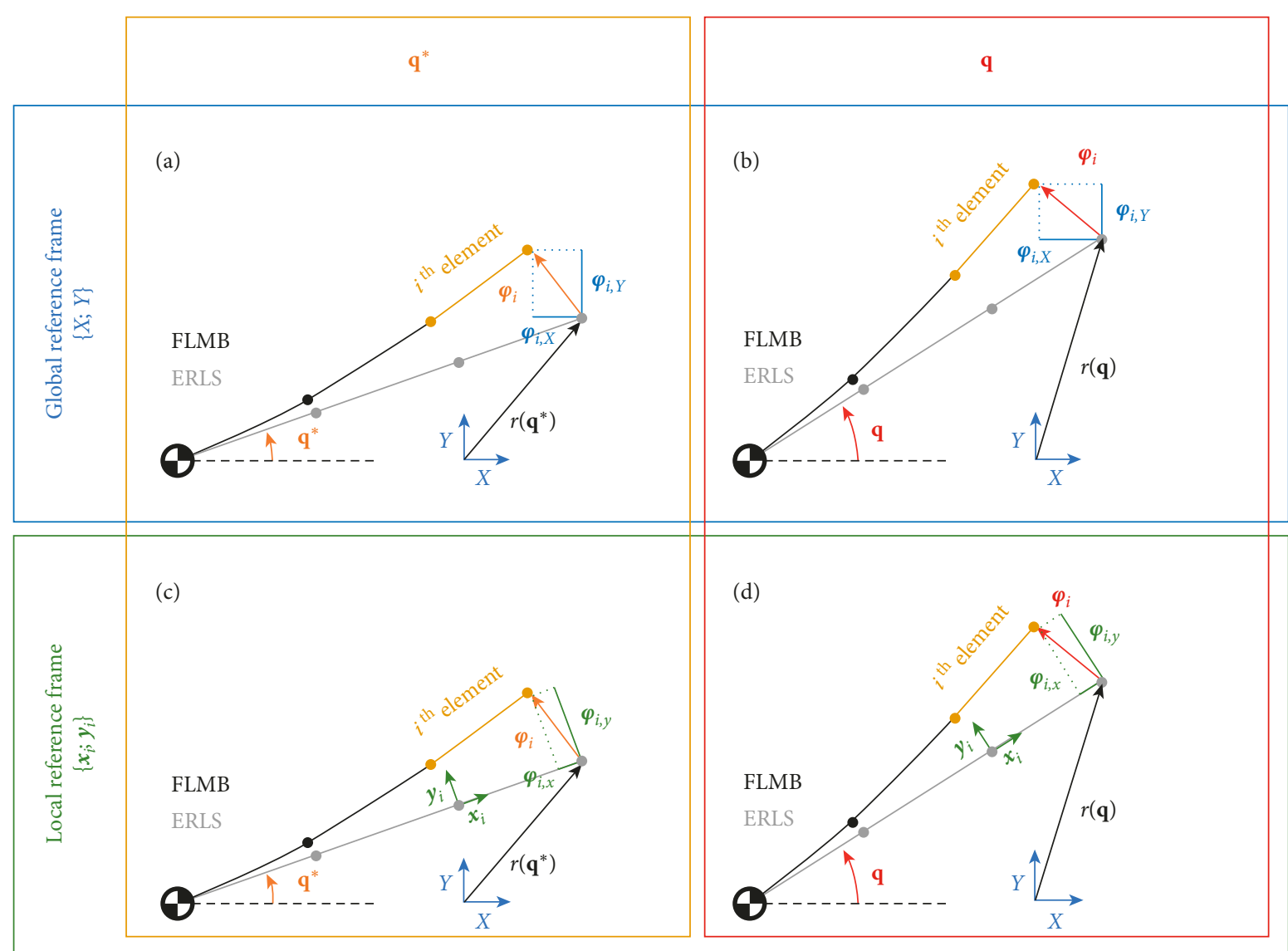

FiguRE 1: Example of eigenvector components in a planar flexible single link for two ERLS configurations: $\mathbf{q}^{*}$ (a-c) and $\mathbf{q}(\mathrm{b}-\mathrm{d}$ ), with respect to the global reference frame $(a-b)$ and the local reference frame (c-d).

3.2. The Extended Kalman Filter (EKF). The estimation algorithm employed in this work is the EKF [25] that is the simplest and most widespread extension of the well-known Kalman filter to nonlinear state estimation. Such a filter requires a discrete-time representation of the system model in Equation (8):

$$
\left\{\begin{array}{l}
\mathbf{z}_{k}=f_{\mathrm{d}}\left(\mathbf{z}_{k-1}, \widetilde{\mathbf{F}}_{k-1}, \mathbf{v}_{k-1}\right), \\
\mathbf{y}_{k}=g\left(\mathbf{z}_{k}, \mathbf{w}_{k}\right),
\end{array}\right.
$$

$f_{\mathrm{d}}$ is the discrete-time state equation, obtained by discretizing $f$ through any arbitrary scheme and $k$ refers to the $k$ th time sample. In Equation (10), $\mathbf{v}_{k-1}$ and $\mathbf{w}_{k}$ are the process noise and measurement noise that are assumed in the Kalman theory to be zero-mean uncorrelated Gaussian noise with covariance matrices $\mathbf{Q}$ and $\mathbf{R}$, respectively.

The EKF is a discrete-time recursive algorithm that operates in two stages: the prediction and the correction. Only a brief explanation is provided here. The readers could refer to the quoted literature for a more detailed description.

In the first stage, at each time step $k$, the filter predicts the state and the observation by means of the nonlinear model and measurement equations, respectively:

$$
\begin{aligned}
& \widehat{\mathbf{z}}_{k \mid k-1}=f_{\mathrm{d}}\left(\widehat{\mathbf{z}}_{k-1 \mid}, \widetilde{\mathbf{F}}_{k-1 \mid}, \mathbf{v}_{k-1 \mid}\right), \\
& \widehat{\mathbf{y}}_{k \mid k-1}=g\left(\widehat{\mathbf{z}}_{k \mid k-1}, \mathbf{w}_{k}\right) .
\end{aligned}
$$

Additionally, it propagates the state and observation error covariance matrices (denoted as $\widehat{\mathbf{P}}_{k \mid k-1}^{\mathbf{Z Z}}, \widehat{\mathbf{P}}_{k \mid k-1}^{\mathbf{y y}}$, and $\widehat{\mathbf{P}}_{k \mid k-1}^{\mathbf{z y}}$ ) by means of the Jacobians of the system and measurement equations ( $\widehat{\mathbf{A}}_{k-1}$ and $\widehat{\mathbf{D}}_{k \mid k-1}$ respectively), computed about the estimated state trajectory:

$$
\begin{aligned}
\widehat{\mathbf{A}}_{k-1} & =\left.\frac{\partial \mathbf{f}}{\partial \mathbf{z}}\right|_{\widehat{\mathbf{z}}_{k-1}, \widetilde{\mathbf{F}}_{k-1}}, \\
\widehat{\mathbf{P}}_{k \mid k-1}^{\mathbf{z z}} & =\widehat{\mathbf{A}}_{k-1} \widehat{\mathbf{P}}_{k-1}^{\mathbf{z z}} \widehat{\mathbf{A}}_{k-1}^{\mathrm{T}}+\mathbf{Q}, \\
\widehat{\mathbf{D}}_{k \mid k-1} & =\left.\frac{\partial \mathbf{g}}{\partial \mathbf{z}}\right|_{\widehat{\mathbf{z}}_{k \mid k-1}}, \\
\widehat{\mathbf{P}}_{k \mid k-1}^{\mathbf{y y}} & =\widehat{\mathbf{D}}_{k \mid k-1} \widehat{\mathbf{P}}_{k \mid k-1}^{\mathbf{z z}} \widehat{\mathbf{D}}_{k \mid k-1}^{\mathrm{T}}+\mathbf{R}, \\
\widehat{\mathbf{P}}_{k \mid k-1}^{\mathbf{z y}} & =\widehat{\mathbf{P}}_{k \mid k-1}^{\mathbf{z z}} \widehat{\mathbf{D}}_{k \mid k-1}^{\mathrm{T}} .
\end{aligned}
$$

Once the next measurements $\mathbf{y}_{k}$ are available, the predicted state is corrected through the error of output estimation $\left(\mathbf{y}_{k}-\widehat{\mathbf{y}}_{k \mid k-1}\right)$ weighed through the filter gain $\mathbf{K}_{k}$ :

$$
\begin{aligned}
\widehat{\mathbf{z}}_{k} & =\widehat{\mathbf{z}}_{k \mid k-1}+\mathbf{K}_{k}\left(\mathbf{y}_{k}-\widehat{\mathbf{y}}_{k \mid k-1}\right), \\
\mathbf{K}_{k} & =\widehat{\mathbf{P}}_{k \mid k-1}^{\mathbf{z y}}\left[\widehat{\mathbf{P}}_{k \mid k-1}^{\mathbf{y y}}\right]^{-1} .
\end{aligned}
$$

Finally, the state error covariance matrix is updated: 


$$
\widehat{\mathbf{P}}_{k}^{\mathbf{z z}}=\widehat{\mathbf{P}}_{k \mid k-1}^{\mathbf{z z}}-\mathbf{K}_{k} \widehat{\mathbf{P}}_{k \mid k-1}^{\mathrm{yy}} \mathbf{K}_{k}^{\mathrm{T}} .
$$

Equations (12) and (13) reveal that gain $\mathbf{K}_{k}$ of the filter correction depends on the covariance matrices $\mathbf{Q}$ and $\mathbf{R}$. A successful estimation relies both on an accurate model and on a good tuning of these matrices: while $\mathbf{R}$ can be measured, $\mathbf{Q}$ should be usually tuned to give the proper weights to model predictions and noisy measurements in state estimates.

\section{Numerical Validation}

Numerical simulations have been performed through Matlab to validate the nonlinear reduced-order observer. Such a validation allows for an effective assessment of the observer outcomes since it is possible to evaluate and compare any state variable, including the elastic ones which are difficult to measure in an experimental setup. Additionally, it is possible to evaluate the impact of the model reduction on the model accuracy and its effect on the observer stability. Indeed, a wrong model reduction causes relevant estimation errors and might lead to instability because of observation spillover phenomena [20].

4.1. System Description. The planar mechanism shown in Figure 1 is used as the test case for the validation. The mechanism lies on the horizontal plane and is supposed to be driven by three motors: two motors drive the absolute rotation of links 1 and 2, while the third one drives the relative rotation between links 4 and 5 . All the links have circular cross section and dimensions as shown in Figure 2(a) and are supposed to be made of aluminium with elastic modulus $69 \mathrm{GPa}$ and mass density $2700 \mathrm{~kg} / \mathrm{m}^{3}$. Twonode, six-dof beam elements have been employed to model the links, leading to the finite element model shown in Figure 2(a). The joints, the brakes, and the rotors of the motors have been modeled as lumped masses and nodal inertias, whose values are stated in Table 1.

The resulting full-order model has 30 dofs, including three ERLS coordinates (defined as the absolute rotations of links 1, 2, and 5) and 27 elastic displacements. The full-order model has been reduced by applying the reduction strategy discussed in Section 2.2 and by selecting the interior modes to be retained by means of the interior mode ranking (IMR) method [25]. Such a method ranks the interior modes based on their contributions to the dynamics of the fullorder system at the frequencies of interest, i.e., to represent the vibrational modes of the full system that have higher controllability and observability and hence mainly affect the system dynamics. By means of a correct representation of these vibrational modes, it is possible to synthesize a reduced-order model, and hence an observer, that accurately represents the system dynamics that are excited and observed. In the test case under investigation, a reduced model accurately matching the full system dynamics in the frequency range $0-180 \mathrm{~Hz}$ has been obtained that is a reasonable value if compared with the typical bandwidths of motion controllers. Indeed, vibrational modes outside the controller bandwidth cannot be neither excited nor controlled by the control loop. To reach such a goal, the interior modes have been ranked trough the IMR method, and then the number of interior modes to be retained has been evaluated through accuracy indices, comparing all the eigenstructure of the full-order model in the frequency range $0-180 \mathrm{~Hz}$ with the ones of the reduced-order model synthesized at the equilibrium configuration $\mathbf{q}^{*}=\left\{\begin{array}{lll}1.0821 & 2.6354 & 4.7123\end{array}\right\}^{T}$. Two indices are used. The first one is normalized cross orthogonality (NCO), which provides a measure of the mass-matrixorthogonality between the vibrational mode of interest represented through the full-order model $\varphi$ and the reduced-order one $\widetilde{\varphi}$ :

$$
\mathrm{NCO}=\frac{\boldsymbol{\varphi}^{T} \overline{\mathbf{M}} \mathbf{H} \tilde{\boldsymbol{\varphi}}}{\left(\boldsymbol{\varphi}^{T} \overline{\mathbf{M}} \boldsymbol{\varphi}\right)\left(\tilde{\boldsymbol{\varphi}}^{T} \mathbf{H}^{T} \overline{\mathbf{M}} \mathbf{H} \tilde{\boldsymbol{\varphi}}\right)} .
$$

The second index is the relative percentage error on the natural frequencies:

$$
\varepsilon_{f}=\frac{|f-\tilde{f}|}{f} \cdot 100 .
$$

In Equation (17), $f$ and $\tilde{f}$ are the frequency of the mode of interest in the full and in the reduced-order model, respectively. The target value for the NCO is one, while it is zero for $\varepsilon_{f}$. By assuming reasonable thresholds on these indices, the number of interior modes to be retained is determined and hence the truncated interior mode matrix $\widetilde{\boldsymbol{\Phi}}$ is determined.

The set of the reduced coordinates obtained in this example has just 13 dofs and contains 8 physical coordinates $\mathbf{x}_{m}$ (i.e., the master dofs, shown in red in Figure 2(b)) and 5 interior modal coordinates $\boldsymbol{\eta}$ :

$$
\begin{aligned}
\mathbf{x}_{m} & =\left\{\begin{array}{llllllll}
\mathbf{q}_{1} & \mathbf{q}_{2} & \mathbf{q}_{3} & \mathbf{x}_{14} & \mathbf{y}_{15} & \boldsymbol{\theta}_{24} & \mathbf{x}_{25} & \mathbf{y}_{26}
\end{array}\right\}^{\mathrm{T}}, \\
\mathbf{\eta} & \in \mathbb{R}^{5 \times 1} .
\end{aligned}
$$

Table 2 shows the values of the NCO and the $\varepsilon_{f}$ for all the vibrational modes of the system in the frequency range of interest at the equilibrium configuration $\mathbf{q}^{*}$. It is evident that the system dynamic is correctly represented. Similar results have been obtained by evaluating the accuracy of such a reduced model in a neighborhood of the equilibrium configuration (see [25] for a detailed description).

Having reduced to 13 the dimensions of the model, the first-order formulation of the reduced model has a $26-\mathrm{di}-$ mensional state vector versus the 60 state variables of the full-order model. Thus, a great reduction has been obtained.

The measured inputs of the state observer are the three torques exerted by the three actuators $\left(T_{1}, T_{2}\right.$, and $T_{3}$ in Figure 2(b)):

$$
\widetilde{\mathbf{F}}=\left\{\begin{array}{lllllllllllll}
T_{1} & T_{2} & T_{3} & 0 & 0 & -T_{3} & 0 & 0 & 0 & 0 & 0 & 0 & 0
\end{array}\right\}^{T} .
$$

Six sensed outputs are available to compute the observer innovation: the angular positions of the three-actuated links $\left(q_{1}, q_{2}\right.$, and $\left.q_{3}\right)$ and the curvatures (strains) at the midpoints of links 1, 2, and 4 (Figure 2(b)), denoted as $\gamma_{1}, \gamma_{2}$, and $\gamma_{4}$ : 


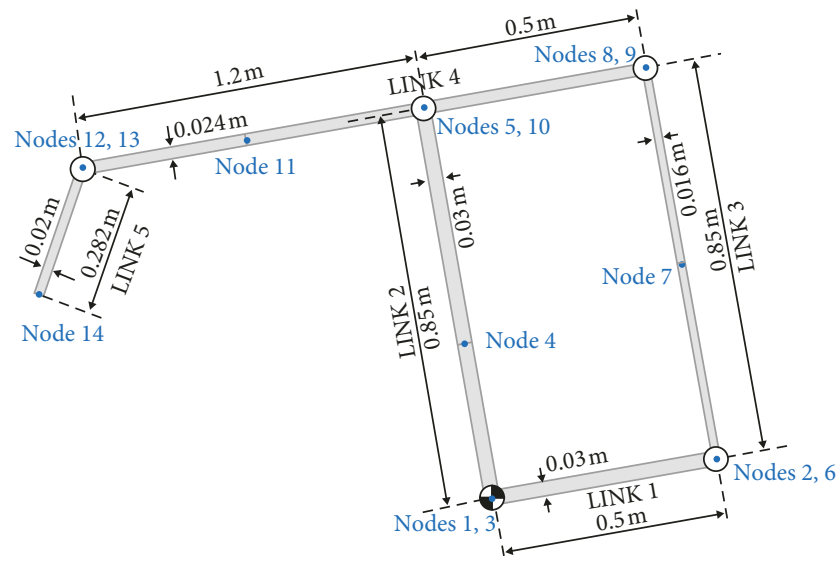

(a)

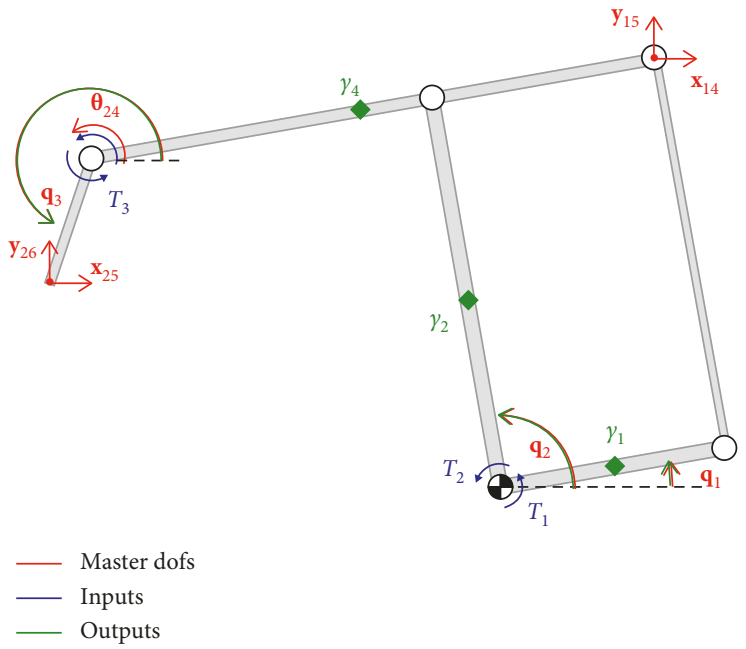

(b)

Figure 2: Studied mechanism: finite element model (a) variables involved in the estimation process (b).

TABLE 1: Lumped masses and nodal inertia.

\begin{tabular}{lcc}
\hline Node & Mass $(\mathrm{kg})$ & Inertia $\left(10^{-2} \mathrm{kgm}^{2}\right)$ \\
\hline 1 & 7.644 & 1.30 \\
2 & 0.392 & 0.00 \\
3 & 9.517 & 2.29 \\
4 & 0.400 & 0.00 \\
5 & 0.648 & 0.00 \\
6 & 0.671 & 0.00 \\
8 & 0.383 & 0.00 \\
9 & 0.658 & 0.00 \\
10 & 0.308 & 0.00 \\
12 & 1.537 & 0.00 \\
13 & 0.095 & 0.15 \\
14 & 0.046 & 0.00 \\
\hline
\end{tabular}

TABLE 2: Evaluation of the accuracy of the reduced model.

\begin{tabular}{lcc}
\hline Mode frequency $(\mathrm{Hz})$ & NCO $(-)$ & $\varepsilon_{f}(\%)$ \\
\hline 13.28 & 1.0000 & 0.0110 \\
43.29 & 1.0000 & 0.0012 \\
64.63 & 1.0000 & 0.0699 \\
124.25 & 0.9937 & 1.7157 \\
143.21 & 0.9983 & 0.5725 \\
159.36 & 1.0000 & 0.0112 \\
\hline
\end{tabular}

$$
\mathbf{y}=\left\{\begin{array}{llllll}
q_{1} & q_{2} & q_{3} & \gamma_{1} & \gamma_{2} & \gamma_{4}
\end{array}\right\}^{T}
$$

It should be noted that $\gamma_{1}, \gamma_{2}$, and $\gamma_{4}$ are nonlinear combination of the state variables. This choice of sensed outputs ensures that system is observable, as corroborated by the rank analysis of the observability matrix obtained by linearizing the model around a set of equilibrium configurations in the whole manipulator workspace.

4.2. Estimation Results. The measurements have been generated through the full-order model, which can be thought of as the "real mechanism." In contrast, the observer is based on the nonlinear reduced-order model and uses noisy measurements since all the simulated measured signals that are fed to observer are supposed to be corrupted by Gaussian white noise with realistic values for the sensors. Such a noise has been generated as normally distributed random numbers (by the "randn" function in MATLAB) having zero mean and the standard deviation (denoted as $\sigma$ ) stated in Table 3 for all the sensed measurements. Then, all the measured signals have been digitized trough a 24-bit analogto-digital converter, with input range $\pm 10 \mathrm{~V}$.

A multirate EKF has been implemented: the continuoustime equations of motion of the real mechanism in Equation (9) have been discretized through the fourth-order Runge-Kutta method with an integration step of $0.1 \mathrm{~ms}$, while the measured signals have been updated at $500 \mathrm{~Hz}$.

A motion lasting 3 seconds has been tested. The manipulator end-effector is required to track a path describing a semicircumference of radius $0.15 \mathrm{~m}$ (shown in Figure 3). The motion of the mechanism is open-loop controlled by the computed torques shown in Figure 4 (computed through the rigid-body model). No closed-loop control of the trajectory is performed, since it is out of the scope of the paper. Additionally, no vibration control is performed in order to excite the vibrational modes and hence to clearly evaluate if the reducedorder model and the observer are able to represent such dynamics. The noisy sensed outputs (Equation (18)) employed for computing the observer innovation are shown in Figure 5.

The estimates of the angular positions and velocities of the three-actuated links are plotted in Figures 6 and 7, respectively, and are compared with those delivered by the real mechanism (i.e., those without noise). The same figures also show the estimation error, i.e., the difference between the two signals. These figures clearly show that the observer is stable, and it delivers accurate estimates of the manipulator gross motion, despite the presence of noise affecting measurements. Indeed, the amplitude of the estimation error is smaller than the measurement noise, as corroborated by the comparison of Figures 4 and 5 . 
TABLE 3: Standard deviations assumed for each measurement noise.

\begin{tabular}{lcccccccc}
\hline $\begin{array}{l}\sigma_{T_{1}} \\
(\mathrm{Nm})\end{array}$ & $\begin{array}{l}\sigma_{T_{2}} \\
(\mathrm{Nm})\end{array}$ & $\begin{array}{l}\sigma_{T_{3}} \\
(\mathrm{Nm})\end{array}$ & $\begin{array}{l}\sigma_{q_{1}} \\
(\mathrm{rad})\end{array}$ & $\begin{array}{c}\sigma_{q_{2}} \\
(\mathrm{rad})\end{array}$ & $\begin{array}{c}\sigma_{q_{3}} \\
(\mathrm{rad})\end{array}$ & $\begin{array}{l}\sigma_{\gamma_{1}} \\
(1 / \mathrm{m})\end{array}$ & $\begin{array}{l}\sigma_{\gamma_{2}} \\
{[1 / \mathrm{m}]}\end{array}$ & $\begin{array}{l}\sigma_{\gamma_{4}} \\
(1 / \mathrm{m})\end{array}$ \\
\hline $2 \cdot 10^{-1}$ & $4 \cdot 10^{-1}$ & $1 \cdot 10^{-3}$ & $3.1 \cdot 10^{-3}$ & $3.5 \cdot 10^{-3}$ & $1 \cdot 10^{-2}$ & $5 \cdot 10^{-5}$ & $8 \cdot 10^{-5}$ & $1.5 \cdot 10^{-4}$ \\
\hline
\end{tabular}

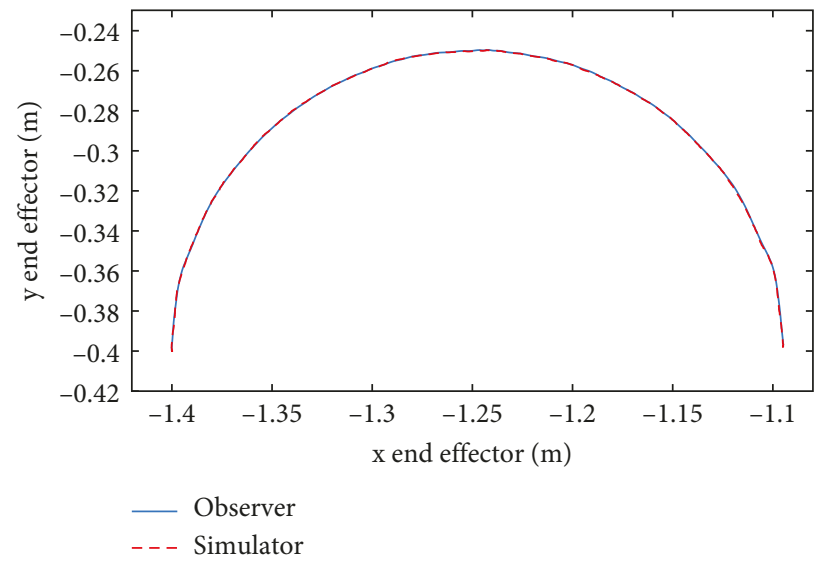

FIGURE 3: End-effector trajectory: actual (red) and estimated (blue).
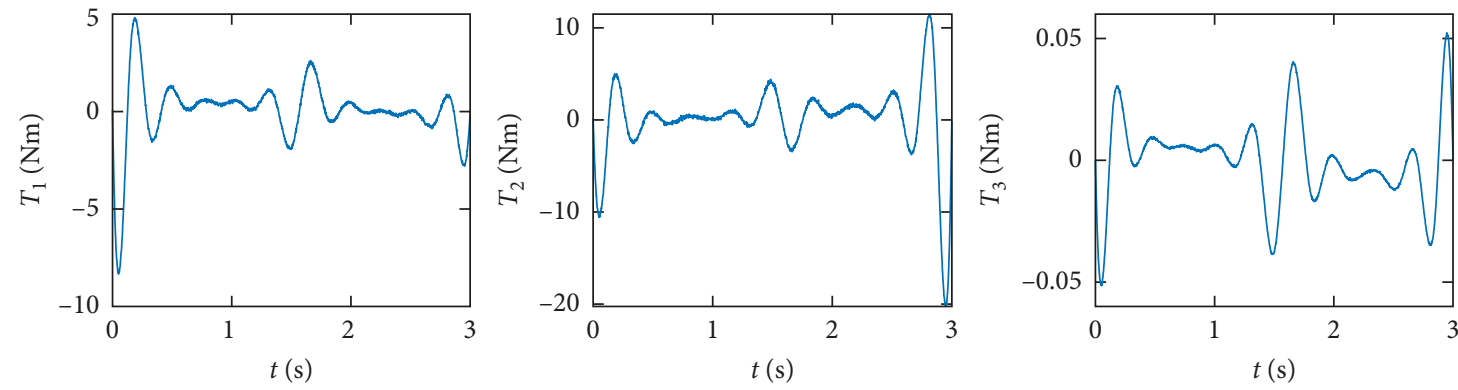

Figure 4: Actuator forces.

Similar considerations can be obtained from Figures 8 and 9, which show the linear elastic displacements and velocities of the manipulator tip that might be of interest for implementing trajectory control schemes. Again, there is a good agreement between the estimated variables and the actual ones, both in terms of amplitude and frequency content of the time-histories.

As a consequence of these precise estimates, the absolute displacement of the manipulator tip is very accurately estimated, and its trajectory in the Cartesian space is almost overlapped to the actual one, as shown in Figure 2.

Further evidences of the observer effectiveness come from the fast Fourier transforms (FFT) of the tip elastic displacements, as shown in Figure 10: the spectra of the estimated ones are almost perfectly overlapped to those of the real mechanism. Such a result further confirms the capability of the reduced-order model to represent correctly the dynamics of the manipulator in the frequency range $0-180 \mathrm{~Hz}$, where the reduced model has been tuned.

4.3. Sensitivity Analysis. A sensitivity analysis has been carried out to assess observer robustness to model uncertainties. In particular, the payload mass carried at the tip of the real mechanism has been modified by increasing it of the $25 \%, 50 \%, 75 \%$, and $100 \%$ of its original value. In contrast, the payload mass of the reduced-order model adopted in the observer has been kept constant and equal to the nominal value. Random measurement noise, as in the previous test, has been adopted to corrupt the measured signals.

Simulation outcomes have been summarized through the mean $\mu$ and the standard deviation $\sigma$ of the estimation errors averaged over 100 simulations for each value of the a payload mass:

$$
\begin{aligned}
& \mu_{\tau}=\frac{1}{100} \sum_{j=1}^{100} \underbrace{1 / n_{\mathrm{s}} \sum_{k=1}^{n_{\mathrm{s}}}\left|\tau_{k, j}-\widehat{\tau}_{k, j}\right|}_{\mu_{j}}, \\
& \sigma_{\tau}=\frac{1}{100} \sum_{j=1}^{100} \sqrt{\frac{1}{n_{\mathrm{s}}-1} \sum_{k=1}^{n_{\mathrm{s}}}\left(\left|\tau_{k, j}-\widehat{\tau}_{k, j}\right|-\mu_{j}\right)^{2} .}
\end{aligned}
$$

In Equation (21), $\tau$ denotes a generic estimated variable; $\tau_{k, j}$ and $\widehat{\tau}_{k, j}$ are the estimated and the actual values of $\tau$, 

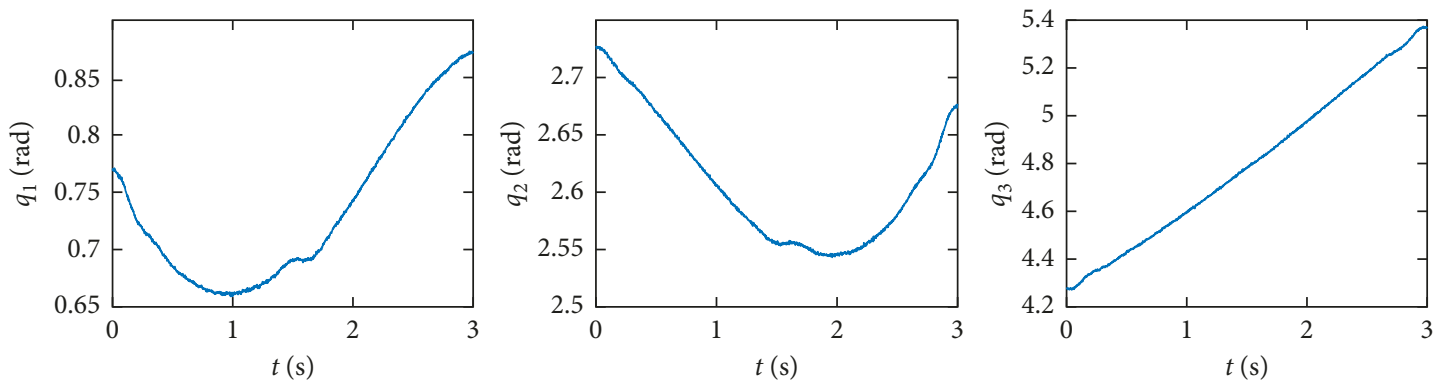

(a)
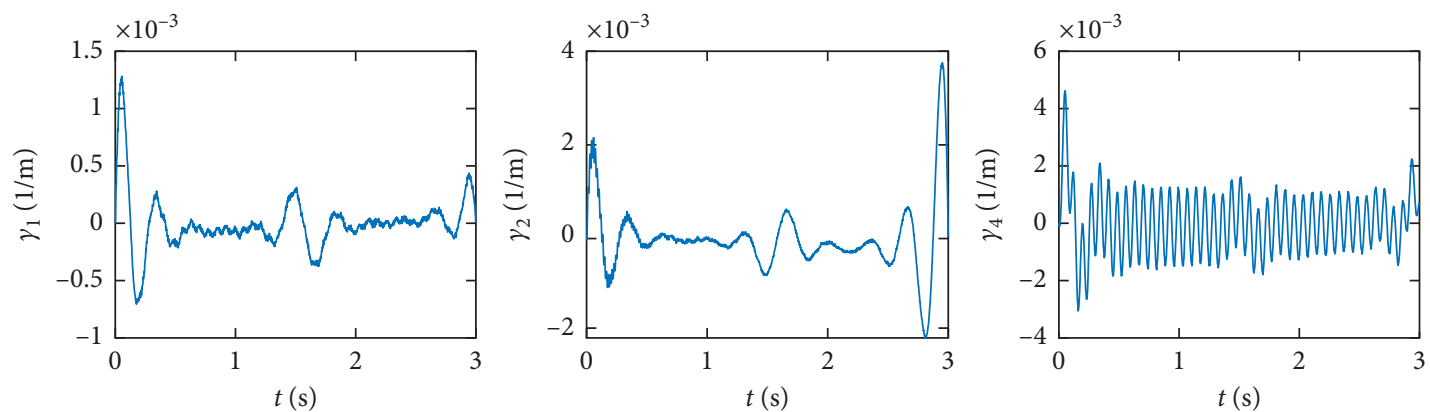

(b)

FiguRE 5: Measured outputs: angular positions of the actuated links (a); curvatures of links 1, 2, and 4 (b).

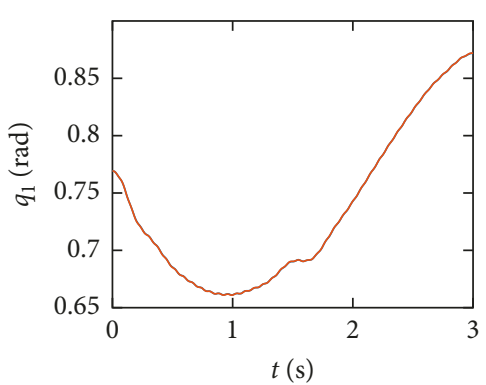

— Observer

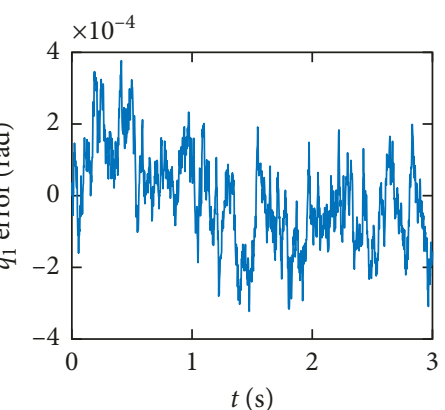

- Observer

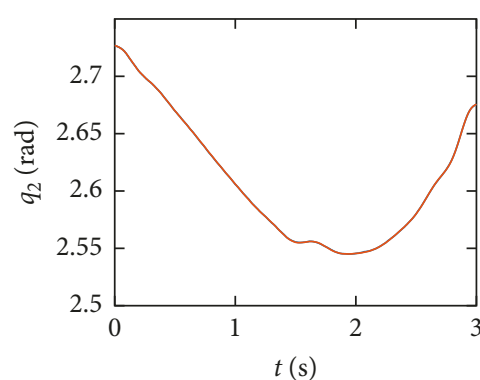

- Observer

- Simulator

(a)

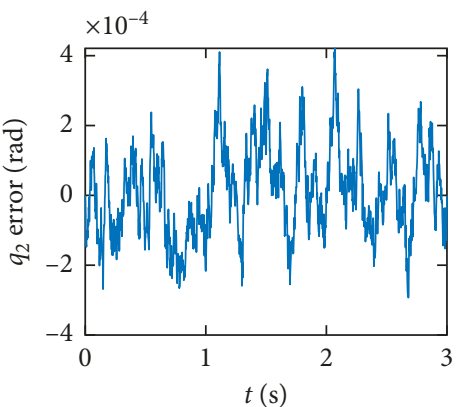

Observer
S Simulator

(b)

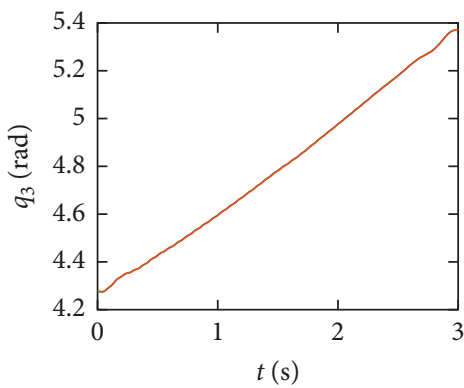

- Observer

— Simulator

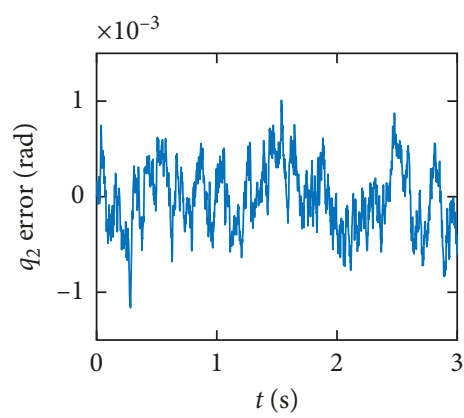

- Observer

_ Simulator

FIgURE 6: Estimated angular positions of the actuated links (a) and estimation errors (b). 

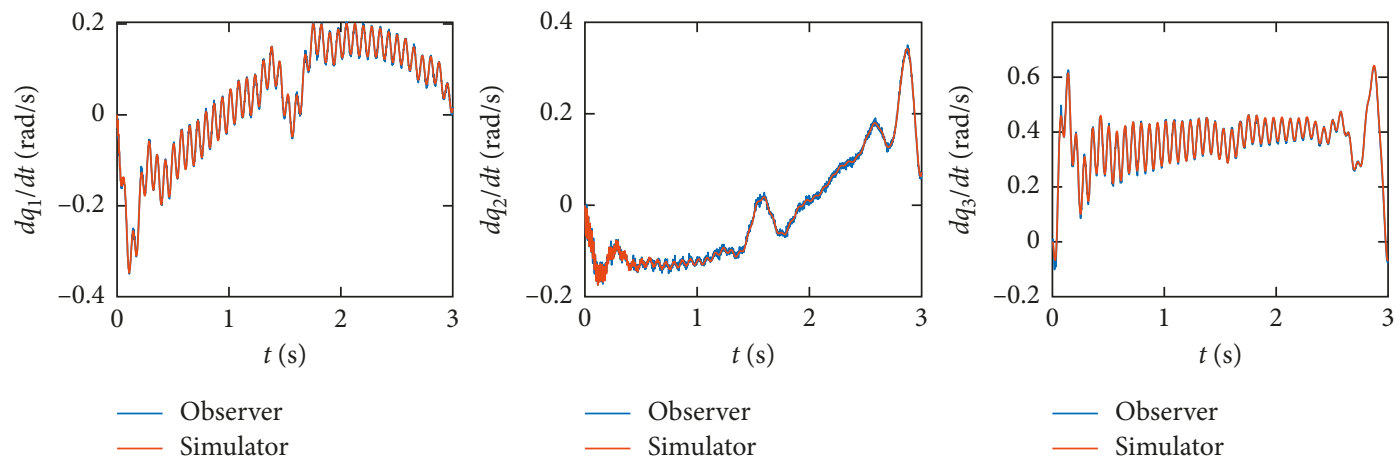

Observer
Simulator

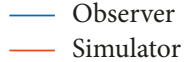

(a)
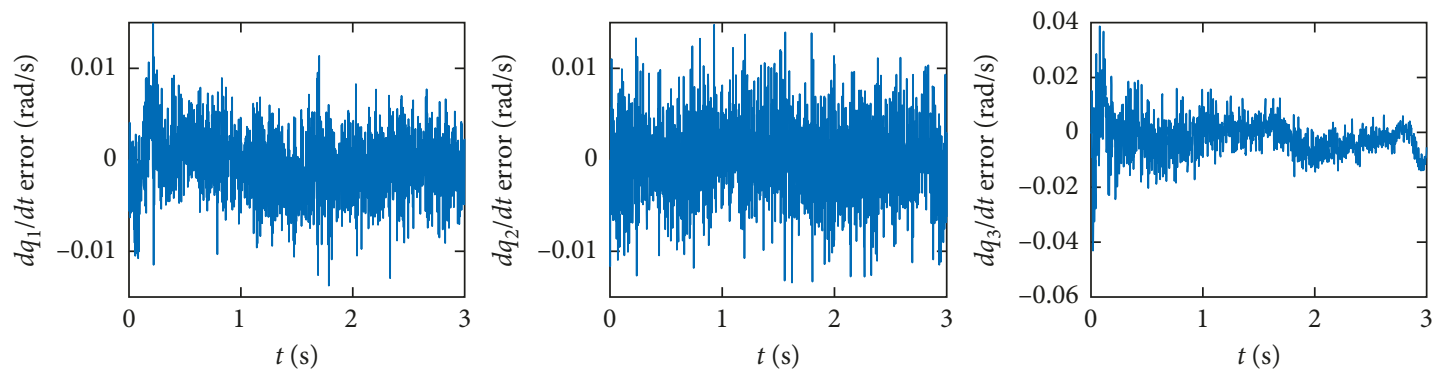

Observer
- Simulator

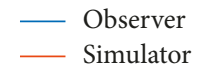

- Observer

(b)

FIGURE 7: Estimated angular velocities of the actuated links (a) and estimation errors (b).
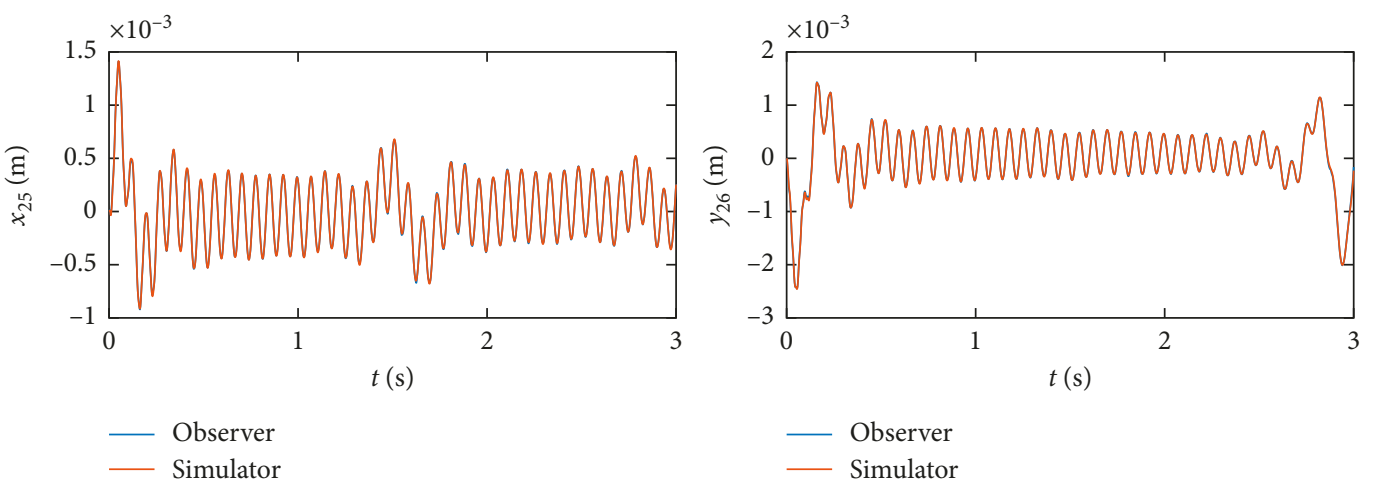

(a)
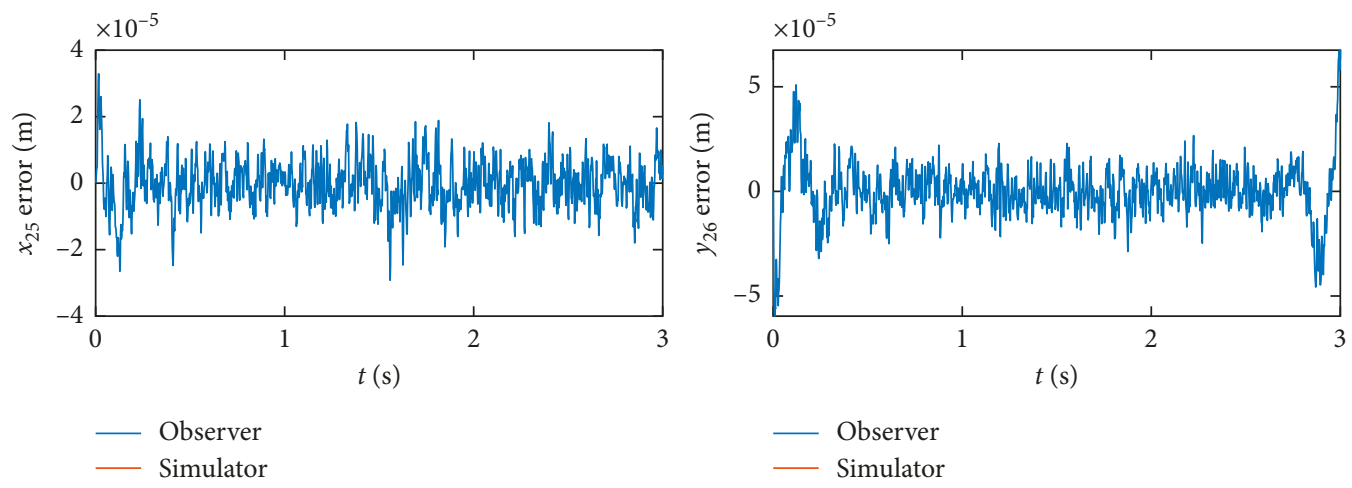

(b)

FIgURE 8: Estimated elastic displacements of the elastic coordinates of the end-effector (a) and estimation error (b). 


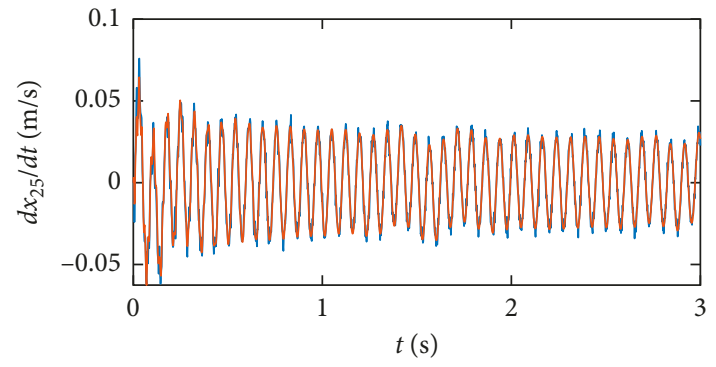

- Observer

— Simulator

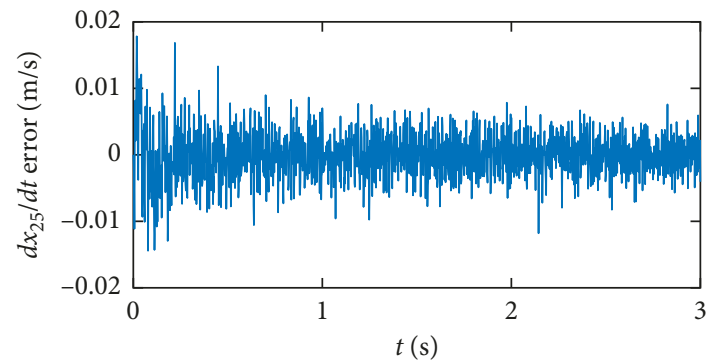

- Observer

Simulator

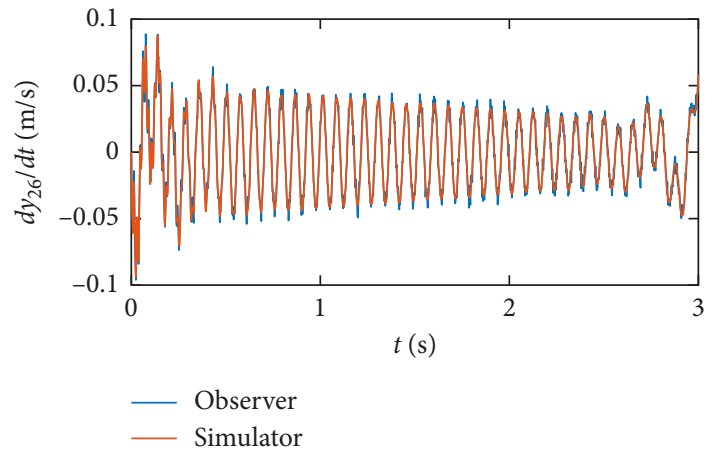

(a)

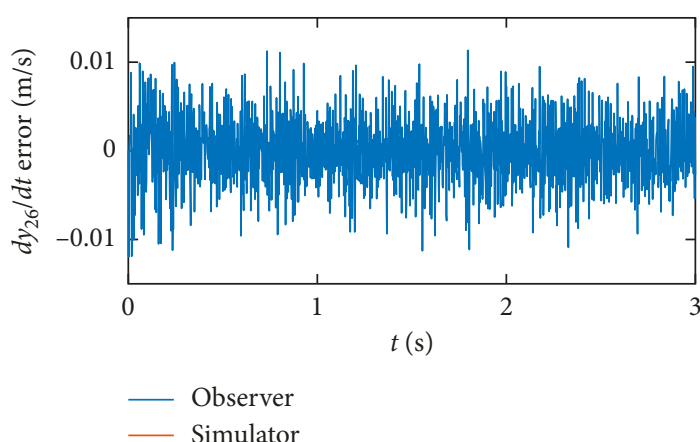

(b)

FiguRE 9: Estimated elastic velocities of the elastic coordinates of the end-effector (a) and estimation error (b).
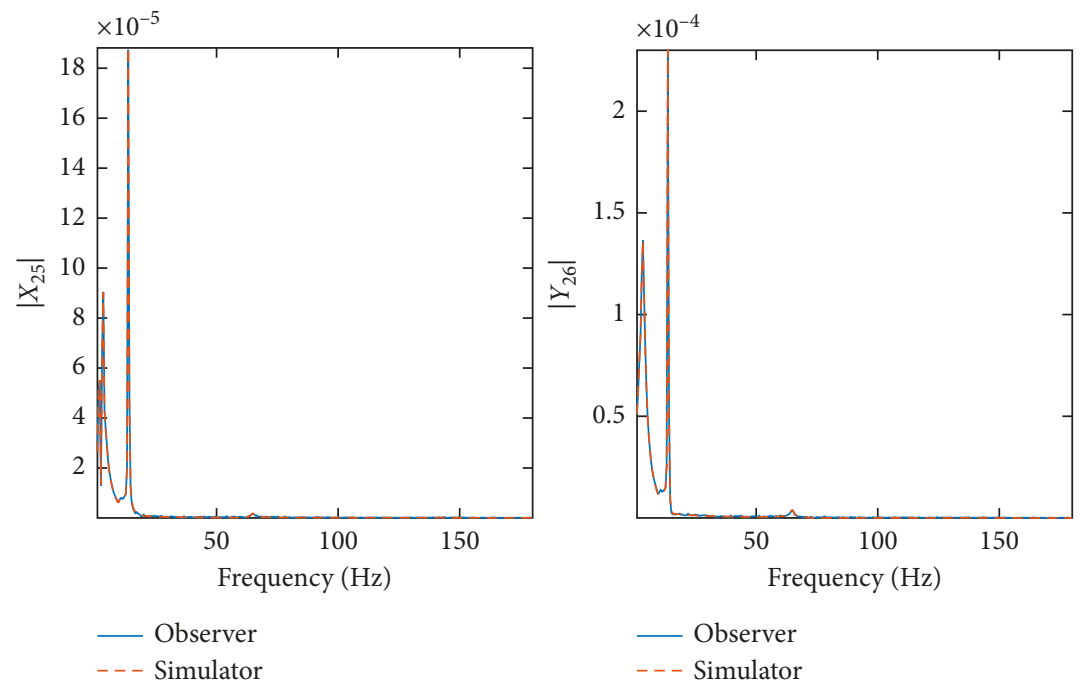

FIGURE 10: FFT of the elastic displacements of the end-effector.

respectively, at the $k$ th simulation step of the $j$ th run; and $n_{\mathrm{s}}$ is the number of sample of each run.

The means (markers) and the standard deviations (lines) of the estimation errors of the linear displacements and velocities of the tip coordinates are shown in Figure 11. Clearly, the case of no added mass corresponds to the results shown in Figures 5-9 for random noise. Although mismodeling affects negatively the estimation by increasing both the mean value and the standard deviation of the errors, the estimation errors are small even in the presence of a payload mass twice the expected one. This result corroborates the correctness of both the model reduction approach and the estimation scheme proposed.

\section{Conclusions}

A nonlinear state observer based on the extended Kalman filter and on a reduced dynamic model of a flexible-link 

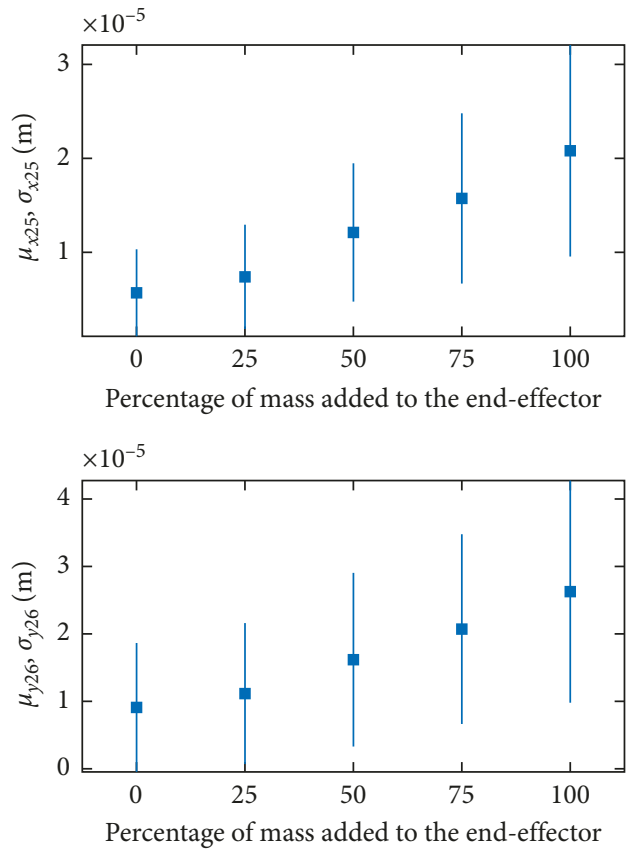

(a)
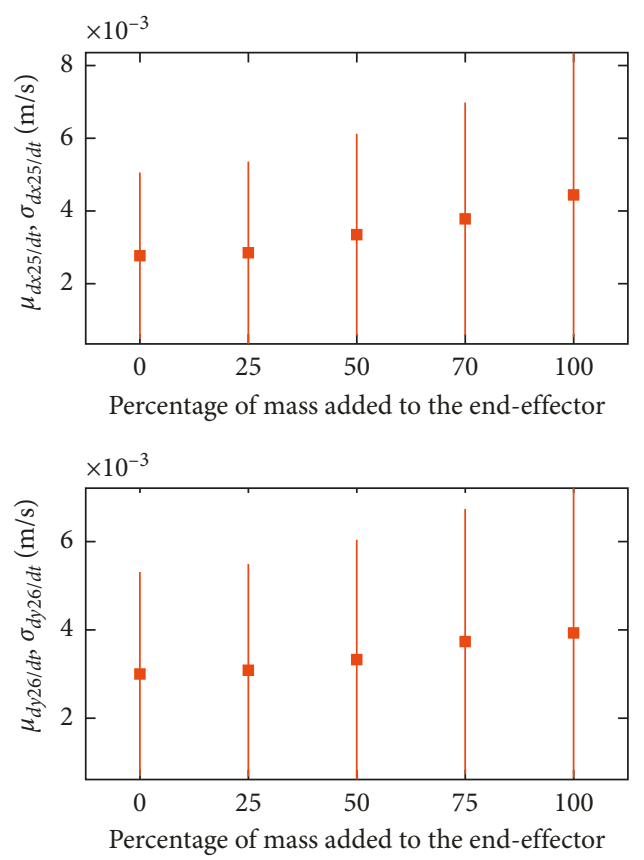

(b)

FIgURE 11: Means (markers) and standard deviations (lines) of the estimation errors on the linear elastic displacements (a) and velocities (b) of the end-effector for different values of payload mass.

manipulator has been successfully synthesized. Model reduction has been performed through a modified CraigBampton strategy that handles nonlinearities by properly modifying the transformation matrix and that trades-off between model size and accuracy through a wise selection of the most important interior modes to be retained.

The numerical results prove that such an observer delivers accurate estimates of both the rigid-body motion and the elastic displacements. Observer robustness to measurement noise and model uncertainties has been also proved by including noise and parameter variation.

The great reduction of the computational effort due to the reduction of the model size makes the proposed observer promising for getting efficient state estimates in the control of flexible-link multibody systems.

\section{Data Availability}

The data used to support the findings of this study are available from the corresponding author upon request.

\section{Conflicts of Interest}

The authors declare that they have no conflicts of interest.

\section{References}

[1] R. Caracciolo, D. Richiedei, and A. Trevisani, "Deformation control in rest-to-rest motion of mechanisms with flexible links," Shock and Vibration, vol. 2018, Article ID 9016028, 9 pages, 2018.

[2] M. Morlock, C. Schröck, M. Burkhardt, and R. Seifried, "Nonlinear state estimation for trajectory tracking of a flexible parallel manipulator," IFAC-PapersOnLine, vol. 50, no. 1, pp. 3449-3454, 2017.

[3] Q. Zhang, X. Zhang, and J. Wei, "Experimental study of active vibration control of planar 3-RRR flexible parallel robots mechanism," Shock and Vibration, vol. 2016, Article ID 4780181, 17 pages, 2016.

[4] E. Lu, W. Li, X. Yang, M. Fan, and Y. Liu, "Modelling and composite control of single flexible manipulators with piezoelectric actuators," Shock and Vibration, vol. 2016, Article ID 2689178, 14 pages, 2016.

[5] E. Barjuei, P. Boscariol, R. Vidoni, and A. Gasparetto, "Robust control of three-dimensional compliant mechanisms," ASME Journal Dynamic Systems, Measurements and Control, vol. 138, no. 10, article 101009, 2016.

[6] H. Ouyang, D. Richiedei, and A. Trevisani, "Pole assignment for control of flexible link mechanisms," Journal of Sound and Vibration, vol. 332, no. 12, pp. 2884-2899, 2013.

[7] M. G. Tehrani and H. Ouyang, "Receptance-based partial pole assignment for asymmetric systems using state-feedback," Shock and Vibration, vol. 19, no. 5, pp. 1135-1142, 2012.

[8] C. Sun, W. He, and J. Hong, "Neural network control of a flexible robotic manipulator using the lumped spring-mass model," IEEE Transactions on Systems, Man, and Cybernetics: Systems, vol. 47, no. 8, pp. 1863-1874, 2017.

[9] Y. Su, P. C. Muller, and C. Zheng, "A simple nonlinear observer for a class of uncertain mechanical systems," IEEE Transactions on Automatic Control, vol. 52, no. 7, pp. 13401345, 2007.

[10] E. Sanjurjo, M. Naya, J. Blanco-Claraco, J. Torres-Moreno, and A. Giménez-Fernández, "Accuracy and efficiency comparison of various nonlinear Kalman filters applied to multibody models," Nonlinear Dynamics, vol. 88, no. 3, pp. 1935-1951, 2017.

[11] E. Sanjurjo, D. Dopico, A. Luaces, and M. A. Naya, "State and force observers based on multibody models and the indirect Kalman filter," Mechanical Systems and Signal Processing, vol. 106, pp. 210-228, 2018.

[12] I. Palomba, D. Richiedei, and A. Trevisani, "Kinematic state estimation for rigid-link multibody systems by means of 
nonlinear constraint equations," Multibody System Dynamics, vol. 40, no. 1, pp. 1-22, 2017.

[13] A. Zuyev and O. Sawodny, "Modelling and control of a shell structure based on a finite dimensional variational formulation," Mathematical and Computer Modelling of Dynamical Systems, vol. 21, no. 6, pp. 591-612, 2015.

[14] P. Makinen, T. Mononen, and J. Mattila, "Inertial sensorbased state estimation of flexible links subject to bending and torsion," in Proceedings of the 14th IEEE/ASME International Conference on Mechatronic and Embedded Systems and Applications, MESA, Oulu, Finland, July 2018.

[15] B. K. Post and W. J. Book, "A robust nonlinear observation strategy for the control of flexible manipulators," in Proceedings-IEEE International Conference on Robotics and Automation, Shangai, China, May 2011.

[16] A. Zuyev and O. Sawodny, "Observer design for a flexible manipulator model with a payload," in Proceedings-45th IEEE Conference on Decision and Control, San Diego, CA, USA, December 2006.

[17] J. I. Medina Martinez, K. Nakano, and S. Umerujan, "Vibration suppression control of a flexible arm using a nonlinear observer with simultaneous perturbation stochastic approximation," Artificial Life and Robotics, vol. 14, no. 4, pp. 539-544, 2009.

[18] Q. Xu, Z. Jing, and S. Hu, "Stability analysis of nonlinear dynamic system with linear observer for a multilink flexible manipulator," International Journal of Non-Linear Mechanics, vol. 103, pp. 27-36, 2018.

[19] R. Caracciolo, D. Richiedei, and A. Trevisani, "Robust piecewise-linear state observers for flexible link mechanisms," Journal of Dynamic Systems, Measurement, and Control, vol. 130, no. 3, article 031011, 2008.

[20] R. Caracciolo, D. Richiedei, and A. Trevisani, "Design and experimental validation of piecewise-linear state observers for flexible link mechanisms," Meccanica, vol. 41, no. 6, pp. 623-637, 2006.

[21] B. Besselink, U. Tabak, A. Lutowska et al., "A comparison of model reduction techniques from structural dynamics, numerical mathematics and system and control," Journal of Sound and Vibration, vol. 332, no. 19, pp. 4403-4422, 2013.

[22] M. Bampton and R. Craig, "Coupling of substructures for dynamic analyses," AIAA Journal, vol. 6, no. 7, pp. 1313-1319, 1968.

[23] R. Vidoni, P. Gallina, P. Boscariol, A. Gasparetto, and M. Giovagnoni, "Modeling the vibration of spatial flexible mechanisms through an equivalent rigid-link system/ component mode synthesis approach," Journal of Vibration and Control, vol. 23, no. 12, pp. 1890-1907, 2017.

[24] S. Boer, R. Aarts, J. Meijaard, D. Brouwer, and J. Jonker, “A two node superelement description for modelling of flexible complex-shared beam-like components," in Proceedings of ECCOMAS Multibody Dynamics, Brussels, Belgium, 2011.

[25] I. Palomba, D. Richiedei, and A. Trevisani, "A reduction strategy at system level for flexible link multibody systems," International Journal of Mechanics and Control, vol. 18, no. 2, pp. 59-68, 2017.

[26] I. Palomba, D. Richiedei, and A. Trevisani, "Mode selection for reduced order modeling of mechanical systems excited at resonance," International Journal of Mechanical Sciences, vol. 114, pp. 268-276, 2016.
[27] M. S. Grewal and A. P. Andrews, Kalman Filtering: Theory and Practice with MATLAB, John Wiley \& Sons, Hoboken, NJ, USA, 2014.

[28] S. M. Kim, J.-G. Kim, K. C. Park, and S.-W. Chae, "A component mode selection method based on a consistent perturbation expansion of interface displacement," Computer Methods in Applied Mechanics and Engineering, vol. 330, pp. 578-579, 2018.

[29] I. Palomba, D. Richiedei, and A. Trevisani, "Energy-based optimal ranking of the interior modes for reduced-order models under periodic excitation," Shock and Vibration, vol. 2015, Article ID 348106, 10 pages, 2015. 


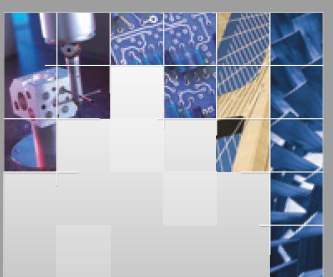

\section{Enfincering}
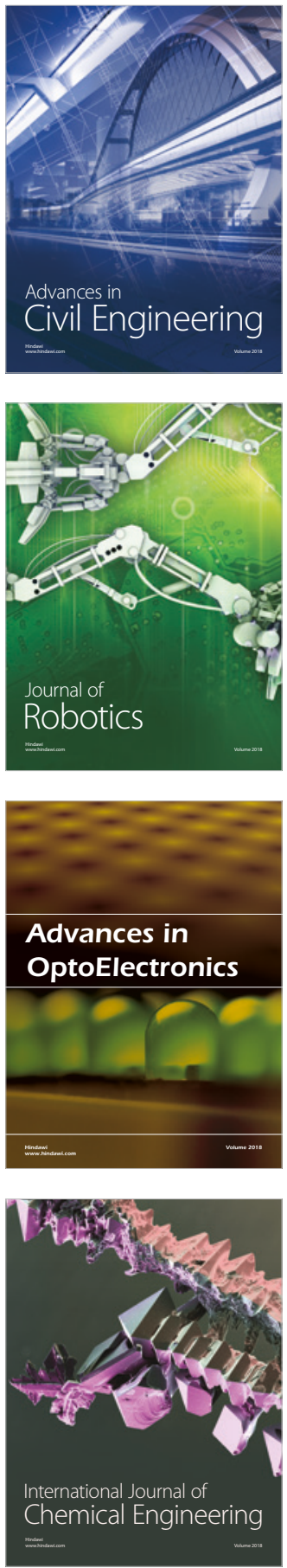

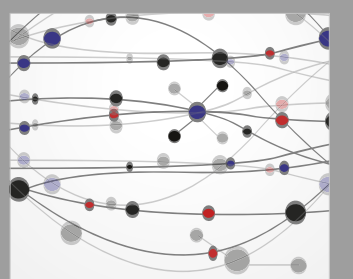

\section{Rotating \\ Machinery}

The Scientific World Journal

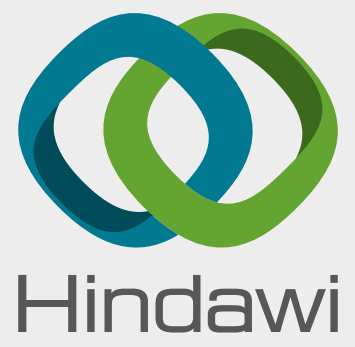

Submit your manuscripts at

www.hindawi.com
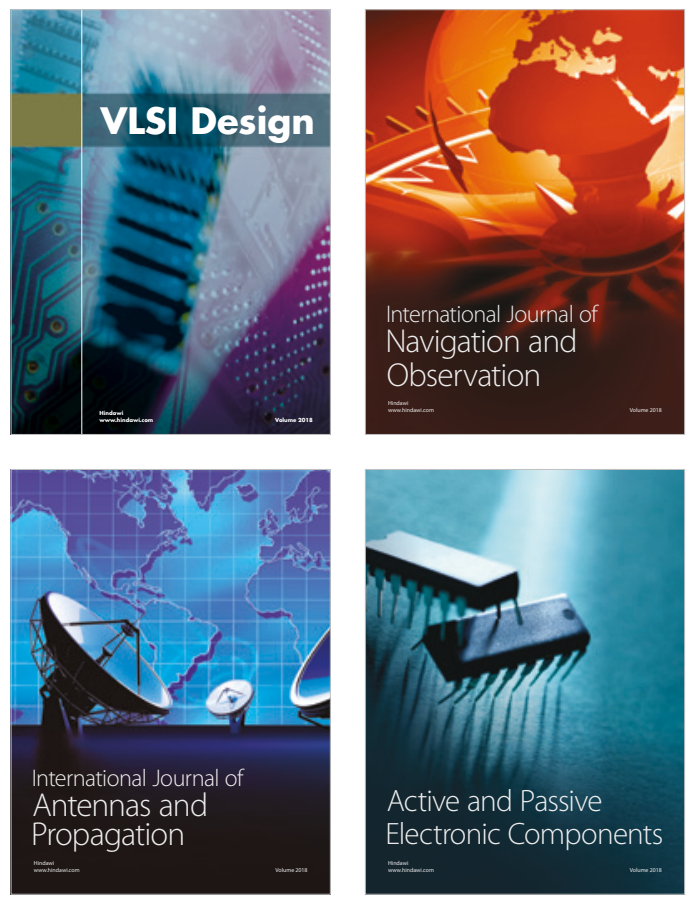
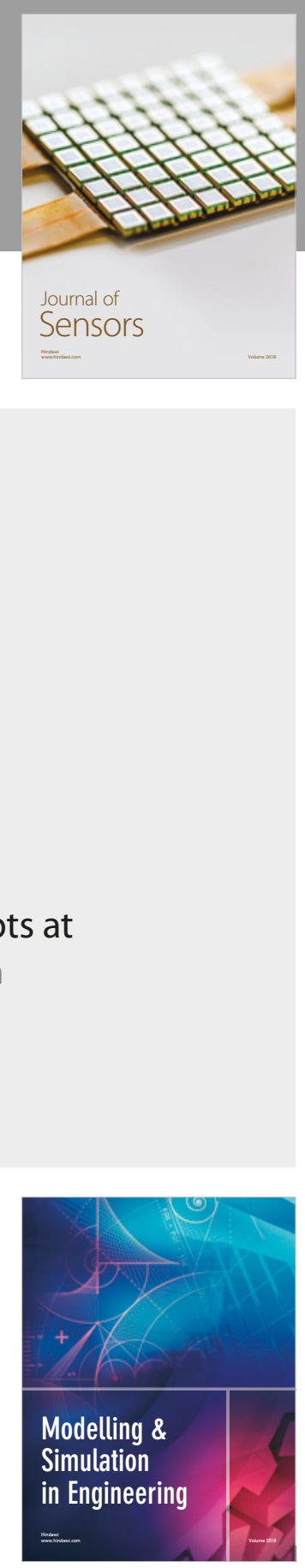

\section{Advances \\ Multimedia}
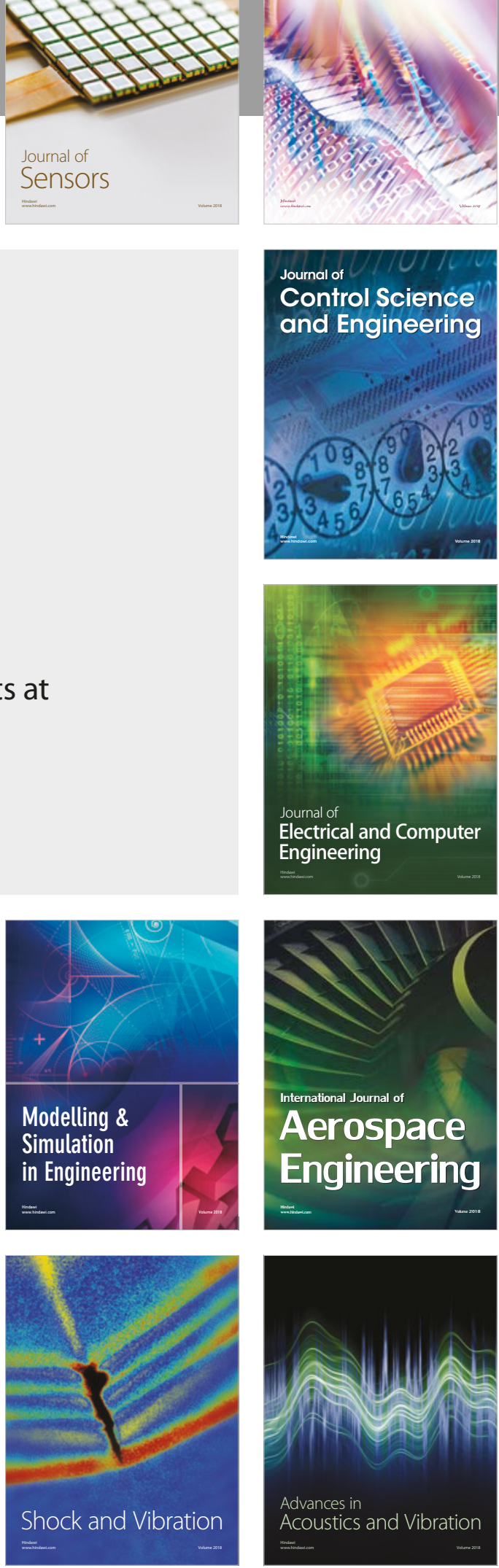